\title{
Collective Phenomena in Multiwall Carbon Nanotubes
}

\author{
M. Margańska*, M. Szopa and E. Zipper \\ Institute of Physics, University of Silesia \\ Uniwersytecka 4, 40-007 Katowice, Poland
}

\begin{abstract}
Collective phenomena due to persistent currents in carbon multiwall nanotubes are studied. The formula for persistent currents minimising free energy and conditions for the stability of persistent currents in multiwall nanotubes in magnetic field are derived. Numerical calculations performed show the possibility of obtaining spontaneous currents in two optimal configurations: undoped armchair-only multiwall nanotubes up to $0.01 \mathrm{~K}$, and zig-zag-chiral-chiral-zig-zag multiwall nanotubes doped to -3.033 eV up to about $1 \mathrm{~K}$. The latter configuration may exhibit also the diamagnetic expulsion of magnetic field, which according to our calculations can reach $20 \%$ of the external flux.
\end{abstract}

PACS numbers: 73.22.-f, 73.23.Ra

\section{Introduction}

It has been indicated [1] that nanoparticles of 2D-layered compounds are inherently unstable in the planar configuration and prefer to form closed structures. This stems from the high energy of the dangling bonds at the periphery of the nanoparticles. In other words, nanotubes and fullerene-like structures form a genuine part of the phase diagram of layered compounds, under the constraint that the crystallite is not allowed to grow beyond a certain size (typically up to a few hundred nanometers). The most famous examples of such structures are carbon nanotubes, but recently many types of inorganic singlewall nanotubes (SWNTs) and multiwall nanotubes (MWNTs), like $\mathrm{MoS}_{2}, \mathrm{WS}_{2}, \mathrm{BN}, \mathrm{V}_{2} \mathrm{O}_{5}$ have been synthesised [2].

On the other hand, it has been shown [3] that if we reduce the size of a cylinder made of normal metal or a semiconductor to mesoscopic dimensions and

*corresponding author; e-mail: magda@phys.us.edu.pl 
if we assume that electrons interact via the magnetostatic interactions, the system exhibits coherent properties absent in macroscopic samples.

In particular, at low $T$ paramagnetic or diamagnetic persistent currents can be induced in such samples by the static magnetic field. If we take into account the current-current (magnetostatic) interaction, persistent current can enhance or shield the external magnetic field and lead to the collective phenomena such as spontaneous flux, flux trapping, and flux expulsion.

In this paper we discuss whether these collective phenomena can be obtained in nanotubes. In order to get these effects we need relatively strong currents. We take as examples single- and multiwall carbon nanotubes, but the presented ideas can be used in the study of inorganic tubes as well.

\section{Interacting persistent currents - collective phenomena}

The persistent current in a system can be derived from the condition that the variation of the free energy with respect to $\phi$ must vanish. While determining its exact form we need to keep in mind the fact that the persistent current running in the system produces an additional magnetic flux

$$
\phi=\phi_{\mathrm{e}}+\mathcal{L} I(\phi),
$$

where $\phi$ is the total flux in the system, $\phi_{\mathrm{e}}$ is the flux introduced by the external magnetic field, $I(\phi)$ is the persistent current, and $\mathcal{L}$ is the self-inductance coefficient which we take to be that of a cylinder

$$
\mathcal{L}=\mu_{0} \frac{\pi R^{2}}{L},
$$

where $R$ is the radius and $L$ - the length of the cylinder. The free energy of the system of $N$ electrons at temperature $T$ is given by

$$
F(T, \phi, N)=-k_{\mathrm{B}} T \sum_{\boldsymbol{k}} \ln \left(1+\exp \left[\left(E_{\boldsymbol{k}}-\mu\right) / k_{\mathrm{B}} T\right]\right)+\frac{\left(\phi-\phi_{\mathrm{e}}\right)^{2}}{2 \mathcal{L}}+N \mu,
$$

where the summation runs over all momentum states $\boldsymbol{k}$. The first term is the usual part of the free energy, the second contains the energy stored in the magnetic field produced by the currents and the last term is necessary to describe a system with a constant number of particles in the grand canonical ensemble [4]. The chemical potential $\mu=\mu(T, \phi, N)$ is calculated from the condition

$$
N=\sum_{k} \frac{1}{1+\exp \left[\left(E_{k}-\mu\right) / k_{\mathrm{B}} T\right]} .
$$

Since the system must be in a stable state, we demand that $\partial F / \partial \phi=0$ at fixed $T$ and $N$

$$
0=\frac{\partial F}{\partial \phi}=\sum_{k} \frac{1}{1+\exp \left[-\left(E_{\boldsymbol{k}}-\mu\right) / k_{\mathrm{B}} T\right]} I_{\boldsymbol{k}}(\phi)-\frac{\partial \mu}{\partial \phi} N+\frac{\phi-\phi_{\mathrm{e}}}{\mathcal{L}}+\frac{\partial \mu}{\partial \phi} N,
$$


where $I_{\boldsymbol{k}}(\phi)=-\partial E_{\boldsymbol{k}} / \partial \phi$ is the current carried at $T=0$ by one momentum state. Using Eq. (1) we obtain the following formula for the persistent current

$$
I(\phi)=\sum_{\boldsymbol{k}} f_{\mathrm{FD}}\left(E_{\boldsymbol{k}}(\phi)\right) I_{\boldsymbol{k}}(\phi)
$$

where $f_{\mathrm{FD}}\left(E_{\boldsymbol{k}}\right)$ is the Fermi-Dirac function. This is the formula we used in our numerical calculations. A simple way of understanding this is to realise that at finite temperatures the weights of states in the Brillouin zone cease to be equal. They are determined by the value of the Fermi-Dirac function for each state and the currents carried by individual states must be multiplied by their FD weights.

In a system consisting of coaxial cylindrical shells, the currents from the individual shells interact. This interaction can lead to interesting effects, like spontaneous flux or flux trapping and flux expulsion, predicted earlier for a mesoscopic cylinder $[3,5]$.

The spontaneous flux can appear in thick cylinders, where all the conducting channels display paramagnetic currents. A slight fluctuation of the magnetic field is then sufficient to cause a persistent current which, in its turn, when the fluctuation disappears, acts against the tendency of the flux to drop to 0 .

The formal derivation of this effect for mesoscopic rings can be found in [6] and applied to our case. The argument goes as follows.

In a multishell system Eq. (1) describing the total flux in the system becomes

$$
\phi_{\mathrm{t}}=\phi_{\mathrm{e}}+\sum_{i} \mathcal{L}_{i} I_{i}\left(\phi_{i}\right)
$$

where $\phi_{\mathrm{e}}$ is the external magnetic flux and the sum over $i$ denotes the summation over all shells of the system. Equation (7) can be written in a way reminiscent of Eq. (1)

$$
\phi_{\mathrm{t}}=\phi_{\mathrm{e}}+\mathcal{L} I_{\mathrm{t}}\left(\phi_{\mathrm{t}}\right)
$$

where the $\left(\sum_{i} \mathcal{L}_{i} I_{i}\left(\phi_{i}\right)\right) / I_{\mathrm{t}}\left(\phi_{\mathrm{t}}\right)$ is approximated by a constant $\mathcal{L}$.

The free energy has now the form

$$
\begin{aligned}
F(T, & \left.\phi_{\mathrm{t}}^{\prime}, N\right)=-k_{\mathrm{B}} T \sum_{i} \sum_{\boldsymbol{k}_{i}} \ln \left(1+\exp \left[\left(E_{\boldsymbol{k}_{i}}-\mu\left(\phi_{i}^{\prime}\right)\right) / k_{\mathrm{B}} T\right]\right) \\
& +\frac{\left(\phi_{\mathrm{t}}^{\prime}-\phi_{\mathrm{e}}^{\prime}\right)^{2}}{2 \mathcal{L}}+N \mu\left(\phi_{\mathrm{t}}^{\prime}\right),
\end{aligned}
$$

where $\phi_{\mathrm{t}}^{\prime}=\phi_{\mathrm{t}} / \phi_{0}$ and $\phi_{\mathrm{e}}^{\prime}=\phi_{\mathrm{e}} / \phi_{0}\left(\phi_{0}=\mathrm{h} / \mathrm{e}\right.$ is the unit flux $)$. We arrive at a relation corresponding in multilayer systems to Eq. (6) for their individual shells

$$
I_{\mathrm{t}}\left(\phi_{\mathrm{t}}\right)=\sum_{i} I_{i}\left(\phi_{i}\right)=\sum_{i} \sum_{\boldsymbol{k}_{i}} \frac{1}{1+\exp \left[\left(E_{\boldsymbol{k}_{i}}-\mu\left(\phi_{i}\right)\right) / k_{\mathrm{B}} T\right]} \frac{\partial E_{\boldsymbol{k}_{i}}}{\partial \phi_{i}} .
$$

The variation of the free energy with respect to $\phi_{\mathrm{t}}^{\prime}$ is 


$$
\frac{\mathrm{d} F}{\mathrm{~d} \phi_{\mathrm{t}}^{\prime}}=-\phi_{0} I_{\mathrm{t}}\left(\phi_{\mathrm{t}}^{\prime}\right)+\frac{\phi_{0}}{\mathcal{L}}\left(\phi_{\mathrm{t}}^{\prime}-\phi_{\mathrm{e}}^{\prime}\right)
$$

and the extremum condition gives

$$
I_{\mathrm{t}}\left(\phi_{\mathrm{t}}^{\prime}\right)=\frac{\phi_{0}}{\mathcal{L}}\left(\phi_{\mathrm{t}}^{\prime}-\phi_{\mathrm{e}}^{\prime}\right)
$$

The equilibrium corresponding to these values of $I_{\mathrm{t}}\left(\phi_{\mathrm{t}}^{\prime}\right)$ can be stable or unstable, depending on the sign of

$$
\frac{\partial^{2} F}{\partial \phi_{\mathrm{t}}^{\prime 2}}=\phi_{0}\left(-\frac{\partial I_{\mathrm{t}}}{\partial \phi_{\mathrm{t}}^{\prime}}+\frac{1}{\mathcal{L}}\right) .
$$

Therefore,

$$
\begin{array}{rlrl}
\text { if } & \frac{\mathrm{d} I_{\mathrm{t}}}{\mathrm{d} \phi_{\mathrm{t}}^{\prime}} & <\frac{1}{\mathcal{L}} \rightarrow \frac{\partial^{2} F}{\partial \phi_{\mathrm{t}}^{\prime 2}}>0 \quad \text { and the equilibrium is stable, } \\
\text { if } & \frac{\mathrm{d} I_{\mathrm{t}}}{\mathrm{d} \phi_{\mathrm{t}}^{\prime}} & >\frac{1}{\mathcal{L}} \rightarrow \frac{\partial^{2} F}{\partial \phi_{\mathrm{t}}^{\prime 2}}<0 & \text { and the equilibrium is unstable. }
\end{array}
$$

Equations (8) and (10) form a set of two self-consistent equations for the current. If their solutions satisfy the relation (14), they are stable and correspond to a minimum of the free energy. This fact implies the existence of a spontaneous current or trapped flux in the system, determined by the intersection of these two functions, and these are what we are searching for. These solutions are shown in Fig. 1.
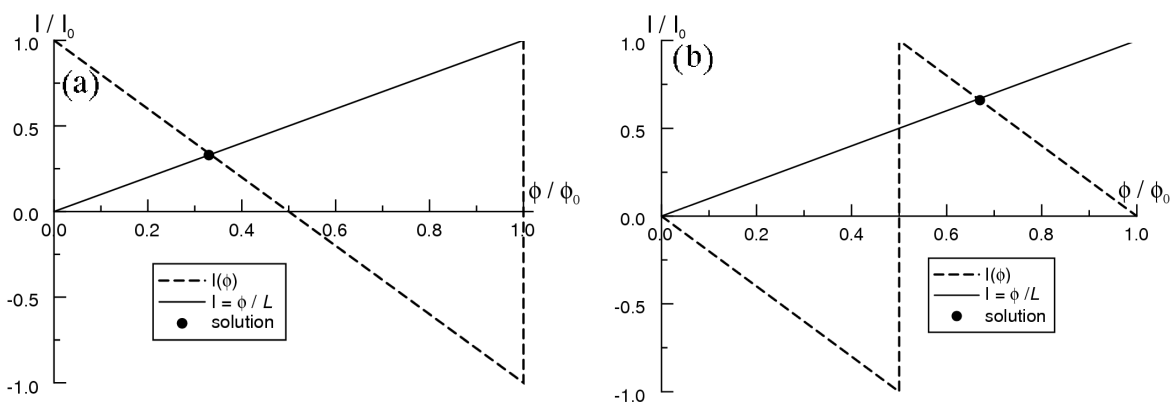

Fig. 1. The solutions of the self-consistent set of Eqs. (8) and (10) in the case of (a) paramagnetic (spontaneous flux) and (b) diamagnetic (flux trapping) current.

In both cases shown in Fig. 1 there is only one stable nonzero solution of the self-consistent set of Eqs. (8) and (10), and it is denoted by the black dot. In a system with a paramagnetic current (a) it corresponds to a spontaneous flux, induced by the slightest fluctuation of the magnetic field. The second solution, at $\phi=0$, is unstable. When the current is diamagnetic (b) this solution is called "trapped flux", since a finite magnetic field must be applied in order to reach this solution. The $I=\phi / \mathcal{L}$ line crosses the $I(\phi)$ also in a second point, but this solution 
is unstable, according to the criterion (14). The solution at $\phi=0$ is stable, but trivial.

\section{Persistent currents and collective phenomena in multiwall carbon nanotubes}

Multiwall carbon nanotubes are nearly ideal examples of the multishell cylindrical structures which we studied in the previous section. Their internal shells can have various chiralities, therefore different currents may run in each shell. Since we obviously aim at systems where spontaneous flux or flux trapping may appear, we need those in which the persistent currents from individual shells both are large and add coherently, either to an overall paramagnetic or diamagnetic current. We have found elsewhere [7] the chirality configurations which yield the largest currents of the optimal shape, and here we only sketch briefly the argument.

In a single shell, stable currents of the largest amplitude can be obtained in metallic undoped nanotubes or in zig-zag nanotubes doped to $-\gamma=3.033 \mathrm{eV}$ [8]. In the former case it is due to the presence of occupied states at the Fermi points, which carry large currents, in the latter - to the specific structure of the Fermi surface, which is flat and parallel to the lines of the constant transverse momentum. The states on one line cross the Fermi surface at the same (integer or half-integer) values of $\phi$ and their currents add coherently as well.

Other chiralities and doping values result in currents which either are by a few orders of magnitude smaller, or have a shape which we are unable to predict.

The persistent current which runs in a metallic undoped shell is sinusoidal, while the current in a zig-zag shell doped to $-\gamma$ has the sawtooth shape characteristic of metallic rings [9]. The current in nanotubes with odd (even) $n$ parameter ${ }^{\dagger}$ corresponds to that in a metallic ring with odd (even) number of electrons, respectively. We are looking for multiwall tubes containing either the greatest number of metallic shells (undoped) or of zig-zag shells of the same parity (doped to $-\gamma$ ), since it is unlikely that different values of doping might be achieved in different shells of the same nanotube (due to inter-layer interactions).

In the latter case it turned out that if the inter-layer spacing of $3.4 \AA$ (close to that in the turbostratic graphite) is to be conserved, the zig-zag shells must be separated by two shells of a different chirality. However, what is more surprising, from the point of view of geometry there is no reason for which a nanotube containing only armchair shells might not be grown. We have therefore chosen for a closer study two sorts of nanotubes: these containing only undoped armchair shells (which we are going to call "A0" configuration) and those containing zig-zag shells divided by two shells of other chiralities, doped to $-\gamma$ (the "Z-1" configuration). The total currents in these two configurations are shown in Fig. 2.

${ }^{\dagger}$ The circumference of the nanotube in the units of the length of lattice generators. 

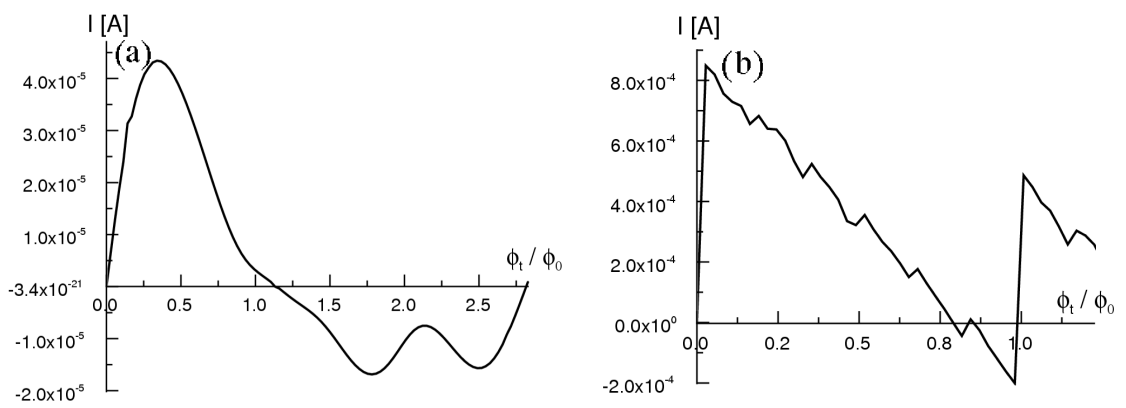

Fig. 2. The superposition of currents from individual shells of a nanotube results in their significant enhancement. Because of the different cross-sections of the constituent shells the periodicity in $\phi$ is lost; (a) the A0 configuration containing 4 shells, with outer diamater of $5 \mathrm{~nm}$ and length of $20 \mathrm{~nm}$, (b) the Z-1 configuration, the same diameter and length, with two active shells.

The fluctuations in the currents are due to the fact that the shells do not all feel the same flux, since their cross-sections are different. For the same reason the current is not periodic in $\phi_{0}$. The more shells contribute to the total current, the smoother is its shape.

Both the large amplitude and shape of the currents make the A0 and Z-1 configurations especially promising as candidates for systems in which collective phenomena might be observed. In the case of the A0 configuration it can only be spontaneous flux, since the persistent currents in metallic tubes are always paramagnetic. The Z-1 configuration can exhibit all the three phenomena, depending on the parity of the zig-zag. In the case of even $n$ it may be the spontaneous flux, in the case of odd $n$ either flux expulsion or flux trapping.

The numerical results of our calculations of spontaneous flux in A0 and even Z-1 configurations are shown in Fig. 3. The spontaneous flux in the configuration $\mathrm{A} 0$ is of the order of $10^{-4} \phi_{0}$ and survives only up to $\approx 0.01 \mathrm{~K}$. The critical temperature in the case of Z-1 configuration is higher, and the spontaneous flux ranges from $\approx 7 \times 10^{-3} \phi_{0}$ at $0.5 \mathrm{~K}$ to $\approx 2 \times 10^{-3} \phi_{0}$ at $1.3 \mathrm{~K}$. In higher temperatures we do not expect spontaneous flux. Since $\mathcal{L}$ is inversely proportional to the length of the tube $L$ and $I(\phi)$ is proportional to it, the term $\mathcal{L} I$ does not depend on the tube length. However, the induced flux is proportional to the nanotube radius, therefore the spontaneous flux might be larger in nanotubes which are wider and thicker.

Since the self-inductance of the nanotubes is usually small, the straight line corresponding to Eq. (8) is very steep and we did not find configurations of nanotube parameters such that the trapping of the flux might be achieved.

Our sample nanotubes have the diamater of $40 \mathrm{~nm}$, which is a little above the upper limit for the commercially grown carbon MWNTs nowadays. Our calculations for the flux expulsion were performed for nanotubes of more optimistic 

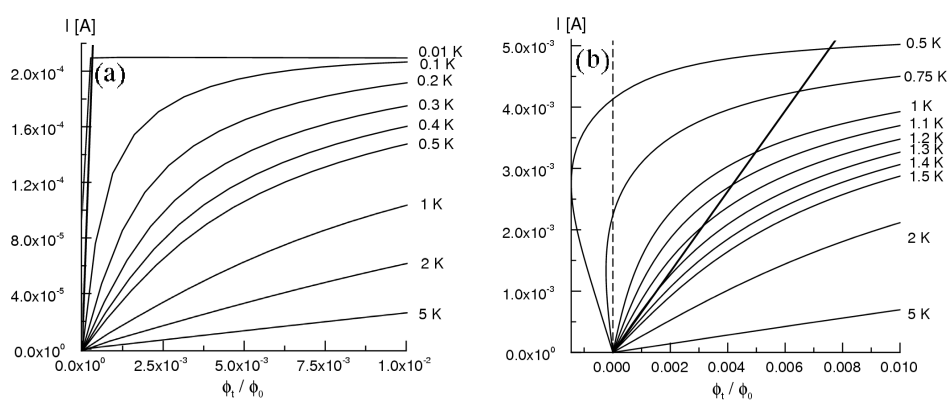

Fig. 3. The total persistent current vs. total flux, for several values of temperature in multiwall carbon nanotubes of inner radius $R_{1}=15 \AA$, outer radius $R_{2}=199 \AA$, and length $L=426 \AA$. These parameters correspond to (a) configuration (A0) with 54 shells (undoped) and (b) configuration Z-1 with 18 active shells (doped to $-\gamma$ ). The straight line in both cases corresponds to Eq. (8). The dashed line in (b) corresponds to $\phi / \phi_{0}=0$.

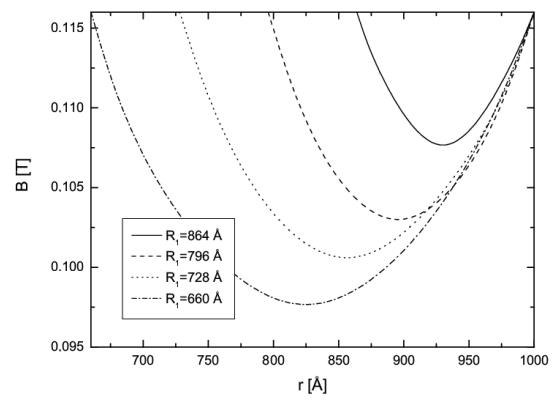

Fig. 4. The flux expulsion in an odd Z-1 multiwall nanotube, with outer radius $R_{2}=$ $100 \mathrm{~nm}$, and the inner $R_{1}=66,73,80$, and $86 \mathrm{~nm}$. The calculations are done for $T=0$.

sizes, that is, about $200 \mathrm{~nm}$ in diameter. Such tubes can be grown [10], but they are so far very dirty and contain topological defects. However, they already exist and the growth techniques can no doubt be refined to rid them of impurities.

The flux expulsion in an odd Z-1 configuration has been calculated using the thick cylinder approach presented in [3] and is shown in Fig. 4. We have fixed the outer radius at $100 \mathrm{~nm}$, varying the inner radius, and therefore the thickness of the tube. The thickest tube contains (again, optimistically) about 100 shells, the next 80,60 , and 40 shells, $1 / 3$ of which is active. The flux expulsion can reach in the case of the thickest nanotube even $20 \%$ of the total flux.

\section{Conclusions}

Our numerical calculations, performed for the MWNT in optimal chirality configurations of the inner shells, show that the spontaneous flux of the order 
of $10^{-4} \phi_{0}$ can be obtained in undoped armchair-only nanotube, with the inner diameter of $3 \mathrm{~nm}$ and outer of $40 \mathrm{~nm}$, but the transition temperature at which this solution vanishes is of the order of $0.01 \mathrm{~K}$.

A similar nanotube but built in zig-zag-chiral-chiral-zig-zag configuration and doped to $-3.033 \mathrm{eV}$ can exhibit fluxes of the order of $10^{-2} \phi_{0}$ up to about $1 \mathrm{~K}$. In the same configuration but with diamagnetic rather than paramagnetic configuration the flux expulsion may appear. Our calculations showed that in a MWNT of the inner radius of $66 \mathrm{~nm}$ and outer of $100 \mathrm{~nm}$ the flux expulsion can reach $20 \%$ of the imposed flux. The flux trapping phenomenon is, according to our calculations, unlikely, due to the small value of self-inductance of a MWNT.

There are obstacles which must be overcome in order to obtain systems with significant persistent currents. Those of technical nature are the improvement of the growth techniques of wide nanotubes, as well as the control over the chiralities of grown shells. The latter seems very difficult to achieve in carbon nanotubes, but it may be easier in inorganic tubes. Using their dispersion relation the calculations analogous to the ones presented here may be performed and the order of spontaneous flux estimated.

\section{References}

[1] Y. Feldman, E. Wasserman, D.J. Srolovitz, R. Tienne, Science 267, 222 (1995).

[2] Carbon Nanotubes - Synthesis, Structure, Properties and Applications, Eds. M.S. Dresselhaus, G. Dresselhaus, P. Avouris, Springer-Verlag, Heidelberg 2001.

[3] M. Czechowska, M. Lisowski, M. Szopa, E. Zipper, Phys. Rev. B 68, 035320 (2003).

[4] F. Reif, Fundamentals of Statistical and Thermal Physics, McGraw-Hill, New York 1965.

[5] M. Stebelski, M. Szopa, E. Zipper, Z. Phys. B 103, 79 (1997).

[6] M. Szopa, E. Zipper Int. J. Mod. Phys. 9, 161 (1995).

[7] M. Szopa, M. Margańska, E. Zipper, M. Lisowski, Phys. Rev. B 70, 075406 (2004).

[8] M. Szopa, M. Margańska, E. Zipper, Phys. Lett. A 299, 593 (2002).

[9] H.-F. Cheung, Y. Gefen, E.K. Riedel, W.-H. Shih, Phys. Rev. B 37, 6050 (1988).

[10] J.-B. Park, G.-S. Choi, Y.-S. Cho, S.-Y. Hong, D. Kim, S.-Y. Choi, J.-H. Lee, K.-I. Cho, J. Cryst. Growth 244, 211 (2002). 